\title{
Drusenoid Pigment Epithelial Detachment: Genetic and Clinical Characteristics
}

\author{
Taiyo Shijo $^{1}$, Yoichi Sakurada ${ }^{1, * \mathbb{D}}$, Koji Tanaka ${ }^{2} \mathbb{D}$, Akiko Miki ${ }^{3} \mathbb{D}$, Seigo Yoneyama ${ }^{1}$, Yumiko Machida ${ }^{2}$, \\ Aya Chubachi ${ }^{3}$, Yu Wakatsuki ${ }^{2}$, Atsushi Sugiyama ${ }^{1}$, Hajime Onoe ${ }^{2}$, Wataru Kikushima ${ }^{1}$, Ryusaburo Mori ${ }^{2}$ \\ and Kenji Kashiwagi ${ }^{1}$ D
}

\section{check for}

updates

Citation: Shijo, T.; Sakurada, Y.; Tanaka, K.; Miki, A.; Yoneyama, S.; Machida, Y.; Chubachi, A.; Wakatsuki, Y.; Sugiyama, A.; Onoe, H.; et al. Drusenoid Pigment Epithelial Detachment: Genetic and Clinical Characteristics. Int. J. Mol. Sci. 2021 22, 4074. https://doi.org/10.3390/ ijms22084074

Academic Editor: Paulino

Gómez-Puertas

Received: 15 March 2021

Accepted: 13 April 2021

Published: 15 April 2021

Publisher's Note: MDPI stays neutral with regard to jurisdictional claims in published maps and institutional affiliations.

Copyright: (c) 2021 by the authors. Licensee MDPI, Basel, Switzerland This article is an open access article distributed under the terms and conditions of the Creative Commons Attribution (CC BY) license (https:// creativecommons.org/licenses/by/ $4.0 /)$
1 Departments of Ophthalmology, Faculty of Medicine, University of Yamanashi, Kofu 400-8510, Japan; tshijoh@yamanashi.ac.jp (T.S.); syoneyama@yamanashi.ac.jp (S.Y.); asugiyama@yamanashi.ac.jp (A.S.); wkikushima@yamanashi.ac.jp (W.K.); kenjik@yamanashi.ac.jp (K.K.)

2 Department of Ophthalmology, Nihon University School of Medicine, Tokyo 173-8610, Japan; tanaka.koji@nihon-u.ac.jp (K.T.); machida.yumiko@nihon-u.ac.jp (Y.M.); wakatsuki.yu@nihon-u.ac.jp (Y.W.); onoe.hajime@nihon-u.ac.jp (H.O.); mori.ryusaburo@nihon-u.ac.jp (R.M.)

3 Department of Surgery, Division of Ophthalmology, Kobe University Graduate School of Medicine, Kobe 650-0017, Japan; acacyey@med.kobe-u.ac.jp (A.M.); achuba@med.kobe-u.ac.jp (A.C.)

* Correspondence: sakurada@yamanashi.ac.jp; Tel.: +81-55-273-9657; Fax: +81-55-273-6757

Abstract: Few studies report drusenoid pigment epithelial detachment (DPED) in Asians. In this multicenter study, we report the clinical and genetic characteristics of 76 patients with DPED, and, for comparison, 861 patients with exudative age-related macular degeneration (AMD) were included. On the initial presentation, the mean best-corrected visual acuity was $0.087 \pm 0.17$ (logMAR unit), and mean DPED height and width were $210 \pm 132$ and $1633 \pm 1114 \mu \mathrm{m}$, respectively. Fifty-one $(67 \%)$ patients showed macular neovascularization in the contralateral eye. The risk allele frequency of both ARMS2 A69S and CFH I62V was significantly higher in DPED than in typical AMD and polypoidal choroidal vasculopathy (PCV) (ARMS2 A69S risk allele frequency: DPED 77\% vs. typical AMD $66 \%$ vs. PCV 57\%, CFH I62V risk allele frequency: DPED 87\% vs. typical AMD 73\% vs. PCV 73\%), although the risk allele frequency of both genes was similar between the DPED group and retinal angiomatous proliferation (RAP) group (ARMS2 A69S: $p=0.32, C F H \mathrm{I} 62 \mathrm{~V}, p=0.11$ ). The prevalence of reticular pseudodrusen (RPD) was highest in RAP (60\%), followed by DPED (22\%), typical AMD $(20 \%)$, and PCV (2\%). Although the prevalence of RPD differs between DPED and RAP, these entities share a similar genetic background in terms of ARMS2 and CFH genes.

Keywords: drusenoid pigment epithelial detachment; ARMS2; CFH; reticular pseudodrusen

\section{Introduction}

Drusenoid pigment epithelial detachment (DPED) is characterized by a fairly wellcircumscribed elevation of the retinal pigment epithelium and a confluence of drusen. The progressive growth and aggregation of large soft drusen is considered to be the origin of DPED, although its pathogenesis is not fully understood.

Casswell et al. described DPED as part of the clinical spectrum of non-exudative age-related macular degeneration (AMD) in 1985 [1]. A recent study using spectral-domain optical coherence tomography (SD-OCT) investigated the quantitative changes in PED and disruption patterns of retinal pigment epithelium (RPE) and Bruch's membrane through the lifecycle of DPED and revealed that maximal volume, height, and diameters of DPED are correlated with visual prognosis [2].

Roquet et al. expanded on previous work and reported that progression to geographic atrophy (GA) or macular neovascularization (MNV) occurred within 2 years if the horizontal width of the DPED was greater than 2 disc diameters on initial presentation [3]. A report from the Age-related Eye Disease Study (AREDS) group found that the 5-year 
incidences of GA and MNV in the natural course of drusenoid PED were 19\% and 23\%, respectively [4].

To date, reports on DPED are almost exclusively seen in Caucasians, and there are few reports investigating the clinical and genetic characteristics of DPED in Asians. In this multicenter study, we genotyped ARMS2 and CFH variants, two major variants associated with AMD, for patients with DPED and investigated the clinical characteristics of DPED at the initial presentation.

\section{Results}

A total of 937 patients, including 76 patients with DPED and 861 patients with exudative AMD, were enrolled in the present study. On the initial presentation, the mean best-corrected visual acuity was $0.087 \pm 0.17$ (logMAR unit), and mean DPED height and width were $210 \pm 132$ and $1633 \pm 1114 \mu \mathrm{m}$, respectively. Of the 76 patients with DPED, genotyping was performed on 64 . Table 1 shows the demographic and genetic characteristics of the participants at the initial presentation. The mean age of the DPED group was similar to that of the typical AMD group. Male distribution in the polypoidal choroidal vasculopathy $(\mathrm{PCV})$ group was the highest among the four groups, followed by the rank order of the Drusenoid PED, typical AMD, and retinal angiomatous proliferation (RAP) groups. Prevalence of reticular pseudodrusen was highest in the RAP group among the four groups, followed by the rank order of the DPED, typical AMD, and PCV groups. The risk allele frequency of ARMS2 A69S was significantly higher in the DPED group than in the typical AMD and PCV groups, although there was no significant difference in the risk allele frequency of ARMS2 A69S between the DPED group and the RAP group. Similar to the risk allele frequency of ARMS2 A69S, the risk allele frequency of $C F H \mathrm{I} 62 \mathrm{~V}$ was significantly higher in the DPED group than in the typical AMD and PCV groups, although there was no significant difference in the risk allele frequency of $C F H \mathrm{I} 62 \mathrm{~V}$ between the DPED group and the RAP group.

Table 1. Demographic and genetic characteristics of drusenoid pigment epithelial detachment and three subtypes of exudative age-related macular degeneration.

\begin{tabular}{|c|c|c|c|c|}
\hline & DPED $(n=76)$ & tAMD $(n=328)$ & PCV $(n=476)$ & $\operatorname{RAP}(n=57)$ \\
\hline Mean age (years) & $77.2 \pm 7.0$ & $76.6 \pm 8.7$ & $73.3 \pm 8.2$ & $82.5 \pm 6.5$ \\
\hline$p$-value (vs. DPED) & & 0.97 & $4.3 \times 10^{-4}$ & $2.3 \times 10^{-5}$ \\
\hline Gender: Male & $42(55.3 \%)$ & $227(69.2 \%)$ & $359(75.4 \%)$ & $23(40.4 \%)$ \\
\hline$p$-value (vs. DPED) & & 0.02 & 0.0003 & 0.09 \\
\hline Presence of RPD & $17(22.4 \%)$ & $65(19.8 \%)$ & $9(1.9 \%)$ & $34(60.0 \%)$ \\
\hline \multirow[t]{2}{*}{$p$-value (vs. DPED) } & & 0.62 & $5.1 \times 10^{-15}$ & $1.2 \times 10^{-5}$ \\
\hline & DPED $(n=64)$ & tAMD $(n=328)$ & PCV $(n=476)$ & $\operatorname{RAP}(n=57)$ \\
\hline \multicolumn{5}{|l|}{$\begin{array}{c}\text { ARMS2 A69S(c.205G > T) } \\
\text { (rs10490924)) }\end{array}$} \\
\hline TT & $38(59.4 \%)$ & $152(46.3 \%)$ & $160(33.6 \%)$ & $39(68.4 \%)$ \\
\hline TG & $23(36.0 \%)$ & $126(38.4 \%)$ & $223(46.8 \%)$ & $16(28.1 \%)$ \\
\hline GG & $3(4.6 \%)$ & $50(15.3 \%)$ & $93(19.6 \%)$ & $2(3.5 \%)$ \\
\hline T-allele frequency & 0.77 & 0.66 & 0.57 & 0.82 \\
\hline$p$-value (vs. DPED) & & $9.2 \times 10^{-3}$ & $7.2 \times 10^{-5}$ & 0.32 \\
\hline \multicolumn{5}{|l|}{$\begin{array}{c}\text { CFH I62V(c.184G > A }) \\
(\text { rs800292) }\end{array}$} \\
\hline GG & $48(75.0 \%)$ & $179(54.6 \%)$ & $259(54.4 \%)$ & $35(61.4 \%)$ \\
\hline GA & $15(23.4 \%)$ & $122(37.2 \%)$ & $176(37.0 \%)$ & $20(35.1 \%)$ \\
\hline AA & $1(1.6 \%)$ & $27(8.2 \%)$ & $41(8.6 \%)$ & $2(3.5 \%)$ \\
\hline G-allele frequency & 0.87 & 0.73 & 0.73 & 0.79 \\
\hline$p$-value (vs. DPED) & & 0.0011 & 0.008 & 0.11 \\
\hline
\end{tabular}

AMD: age-related macular degeneration; DPED: drusenoid pigment epithelial detachment; PCV: polypoidal choroidal vasculopathy; RAP: retinal angiomatous proliferation; RPD: reticular pseudodrusen. 
Table 2 shows the demographic and genetic characteristics of DPED with or without reticular pseudodrusen at the initial presentation. Baseline DPED height was significantly higher in the RPD (-) group than in the RPD (+) group.

Table 2. Comparison of demographic and genetic characteristics between drusenoid pigment epithelial detachment with or without reticular pseudodrusen.

\begin{tabular}{cccc}
\hline & RPD $(+)(n=17)$ & RPD(-) $(n=59)$ & $p$-Value \\
\hline Mean age (years) & $77.65 \pm 7.47$ & $77.19 \pm 6.96$ & 0.78 \\
Gender: Male & $8(47.1 \%)$ & $34(57.6 \%)$ & 0.44 \\
Baseline VA (LogMAR) & $0.11 \pm 0.22$ & $0.082 \pm 0.16$ & 0.80 \\
Presence of MNV in the contralateral eye & $14(82.4 \%)$ & $37(62.7 \%)$ & 0.11 \\
DPED height $(\mu \mathrm{m})$ & $151.12 \pm 52.44$ & $227.24 \pm 142.49$ & 0.028 \\
DPED width $(\mu \mathrm{m})$ & $1339.77 \pm 1182.09$ & $1718.05 \pm 1094.18$ & 0.12 \\
\hline & $\mathbf{R P D}(+)(\boldsymbol{n}=\mathbf{1 6})$ & $\mathbf{R P D}(-)(\boldsymbol{n}=48)$ & $p$-Value \\
\hline ARMS2 A69S(c.205G > T) & & & \\
(rs10490924) & $12(75.0 \%)$ & $26(54.2 \%)$ & \\
TT & $4(25.0 \%)$ & $19(39.6 \%)$ & \\
TG & $0(0.0 \%)$ & $3(6.2 \%)$ & 0.11 \\
GG & 0.88 & 0.74 & \\
T-allele frequency & & & \\
CFH I62V (c.184G $>$ A) & $12(75.0 \%)$ & $36(75.0 \%)$ & \\
(rs800292) & $3(18.8 \%)$ & $12(25.0 \%)$ & \\
GG & $1(6.2 \%)$ & $0(0.0 \%)$ & 0.65 \\
GA & 0.84 & 0.88 & \\
AA & &
\end{tabular}

DPED: drusenoid pigment epithelial detachment; MNV: macular neovascularization; RPD: reticular pseudodrusen.

Table 3 shows the demographic and genetic characteristics of DPED between the MNV $(+)$ and MNV (-) groups at the initial presentation. Baseline DPED height was significantly higher in the MNV (-) group than in the MNV (+) group.

Table 3. Comparison of demographic and genetic characteristics between drusenoid pigment epithelial detachment with or without macular neovascularization in the contralateral eye.

\begin{tabular}{|c|c|c|c|}
\hline & $\operatorname{MNV}(+)(n=51)$ & $\operatorname{MNV}(-)(n=25)$ & $p$-Value \\
\hline Mean age (years) & $77.43 \pm 6.96$ & $76.84 \pm 7.30$ & 0.74 \\
\hline Gender: Male & $27(53.0 \%)$ & $15(60.0 \%)$ & 0.56 \\
\hline Baseline VA (LogMAR) & $0.083 \pm 0.18$ & $0.095 \pm 0.17$ & 0.56 \\
\hline Presence of RPD & $14(27.5 \%)$ & $3(12 \%)$ & 0.13 \\
\hline DPED height $(\mu \mathrm{m})$ & $183.50 \pm 112.43$ & $264.72 \pm 152.12$ & 0.0018 \\
\hline \multirow[t]{2}{*}{ DPED width $(\mu \mathrm{m})$} & $1520.63 \pm 1138.50$ & $1863.56 \pm 1058.77$ & 0.086 \\
\hline & $\operatorname{MNV}(+)(n=48)$ & $\operatorname{MNV}(-)(n=16)$ & $p$-Value \\
\hline \multicolumn{4}{|l|}{$\begin{array}{c}\text { ARMS2 A69S(c.205G > T) } \\
\text { (rs10490924)) }\end{array}$} \\
\hline TT & $31(64.6 \%)$ & $7(43.8 \%)$ & \\
\hline TG & $14(29.2 \%)$ & $9(56.2 \%)$ & \\
\hline GG & $3(6.2 \%)$ & $0(0.0 \%)$ & \\
\hline T-allele frequency & 0.79 & 0.72 & 0.39 \\
\hline \multicolumn{4}{|l|}{$\begin{array}{c}\text { CFH I62V(c.184G > A) } \\
\text { (rs800292) }\end{array}$} \\
\hline GG & $37(77.1 \%)$ & $11(68.8 \%)$ & \\
\hline GA & $10(20.8 \%)$ & $5(31.2 \%)$ & \\
\hline AA & $1(2.1 \%)$ & $0(0.0 \%)$ & \\
\hline G-allele frequency & 0.88 & 0.84 & 0.65 \\
\hline
\end{tabular}

DPED: drusenoid pigment epithelial detachment; MNV: macular neovascularization $n$. 
Of the 51 patients with MNV in the contralateral eye, the prevalence of typical AMD, PCV, RAP, and scarring was $23(45.1 \%), 9(17.6 \%), 14(27.5 \%)$, and $5(9.8 \%)$, respectively.

\section{Discussion}

In this multicenter study, we reported the clinical and genetic characteristics of Japanese patients with DPED. To the best of our knowledge, this is the largest study investigating DPED in Asians.

Yu et al. recently reported that $42.5 \%$ of patients with AREDS2 were male and the mean age was 71.6 years [5]. In the present study, $55 \%$ were male and the mean age was 77.2 years. This might be due to racial differences, including retinal pigment epithelial pigmentation and integrity.

It has been reported that $A R M S 2$ and $C F H$ are two major genes susceptible to agerelated macular degeneration (AMD) through genome-wide association studies [6,7]. Several clinic-based studies have demonstrated that these genes are associated with exudative AMD, including typical AMD, polypoidal choroidal vasculopathy, and retinal angiomatous proliferation (RAP), although there have been no reports investigating the association between DPED and these genes [8,9]. In the present study, we compared the genetic characteristics of DPED with those of the three subtypes of exudative AMD and revealed that DPED and RAP share genetic characteristics in terms of ARMS2 and CFH. Seddon et al. reported that the ARED severity scale increased by increasing the number of risk alleles in ARMS2 and CFH [10]. DPED is a confluence of large soft drusen, and RAP exhibits large soft drusen in the macula [11]. Clinically, they share a fundus appearance. This might be one of the reasons why the risk allele frequencies of both genes were similar.

Reticular pseudodrusen (RPD), also known as subretinal drusenoid deposits, is a distinct entity from conventional drusen [12]. RPD has been reported to be associated with RAP and geographic atrophy. Alten et al. investigated 204 eyes with both PED and RPD and reported that the prevalence of DPED was $24 \%$ [13]. In the present study, RPD was observed in 17 (22\%) of 76 patients with DPED. The prevalence was almost the same as that of typical AMD. To the best of our knowledge, this is the first report regarding the prevalence of RPD in eyes with DPED.

Of the 76 patients with DPED, $51(67 \%)$ showed macular neovascularization (MNV) in the contralateral eye. However, there were no differences between the MNV (+) and MNV $(-)$ groups, except for DPED height at the initial presentation. AREDS 2 did not report the prevalence of DPED depending on the absence or presence of MNV in the contralateral eye. Therefore, we could not compare the present results with those of other studies.

In the present study, we genotyped the ARMS2/CFH variants that were associated with inflammation of the RPE/choroid complex through complement pathway. However, the pathogenesis of AMD is various, such as angiogenesis, mitochondria, and ionchannel [14-16]. Further studies would be needed to clarify the different phenotypes among AMD patients.

The limitations of the study should be mentioned. The first limitation is that it was retrospective in nature; therefore, genotyping was not performed for all patients. The second limitation is that it was a cross-sectional analysis. In the current study, DPED height was lower in the RPD (+) group than in the RPD (-) group. A longitudinal study is necessary to confirm these results.

In summary, we described the clinical and genetic characteristics of DPED in Japanese patients. In particular, DPED and RAP share genetic backgrounds in terms of ARMS2 and $\mathrm{CFH}$, although the prevalence of RPD differs between the two entities.

\section{Methods}

\subsection{Patients}

The medical charts of consecutive patients with drusenoid pigment epithelial detachment at the University of Yamanashi Hospital, Nihon University Hospital, and Kobe University Hospital between January 2012 and December 2020 were used to compare the 
clinical and genetic characteristics between drusenoid PED and exudative AMD; patients with exudative AMD were enrolled at the University of Yamanashi between January 2012 and December 2020. The present study was approved by the institutional review board of each institute and was conducted in accordance with the tenets of the Declaration of Helsinki.

\subsection{Diagnosis}

At the initial presentation, all participants underwent a comprehensive ophthalmic examination, including measurement of best-corrected visual acuity (BCVA) using a Landolt chart, intraocular pressure (IOP), slit-lamp biomicroscopy with a +78-diopter (D) lens, color fundus photography, and spectral domain optical coherence tomography (SD-OCT) examination (Spectralis, Heidelberg Engineering, Dossenheim, Germany, HRA + OCT). If exudation was seen in the contralateral eye, fluorescein angiography (FA) and indocyanine green angiography (ICGA) were also performed.

DPED was defined as a confluence of drusen of more than $500 \mu \mathrm{m}$ in diameter and $100 \mu \mathrm{m}$ in height on SD-OCT, as previously defined [17]. Depending on the presence or absence of MNV in the contralateral eye, patients with DPED were subdivided into the MNV (-) or MNV (+) groups. If there was no exudation in either eyes, these patients were included in the MNV (-) group. A representative case of the MNV (-) group is shown in Figure 1. If exudation occurred in the contralateral eye, these patients were included in the MNV (+) group. A representative case of the MNV (+) group is shown in Figure 2.

Exudative AMD was divided into three subtypes: typical neovascular AMD, PCV, and RAP, which were diagnosed based primarily on FA, ICGA, and SD-OCT findings. Eyes with typical neovascular AMD exhibited classic- or occult-type neovascularization in FA without polypoidal lesions on ICGA and SD-OCT findings of MNV either in the subretinal space or beneath the retinal pigment epithelium line. Eyes with PCV exhibited clusters of polypoidal dilation of the vessels with or without abnormal vascular networks in the superficial choroid in ICGA and irregularly elevated the RPE line on SD-OCT images. Eyes with RAP exhibited retinal-retinal or retinochoroidal anastomosis on FA or ICGA and retinal swelling with or without RPE detachment on SD-OCT, as previously reported [18].

The presence or absence of reticular pseudodrusen (RPD) was assessed using multimodal imaging, including color fundus photography, near-infrared reflectance, fundus autofluorescence, and SD-OCT. We defined the presence of RPD as confirmed by at least one imaging modality, as previously reported [19].

\subsection{Genotyping}

We genotyped two major variants, ARMS2 A69S(rs10490924) and CFH I62V(rs800292), associated with AMD in the Japanese population. On initial presentation, peripheral blood was collected for genomic DNA analysis. The methods used to genotype variants of ARMS2 A69S and CFH I62V have been previously described [20-22]. In detail, TaqMan genotyping assays contain sequence-specific primers to amplify the polymorphic sequence of the target genes and two minor groove binders to stabilize the samples. Purified wet genomic DNA was mixed with TaqMan genotyping assay and dispensed to a reaction plate, and the genotyping with real-time PCR system was performed. The allelic discrimination plot was collected and analyzed and recorded on an anonymous basis. Written informed consent was obtained from all patients.

\subsection{Statistical Analysis}

Statistical analyses were performed using SPSS (IBM, Tokyo, Japan). Best-corrected visual acuity measured on a decimal scale using a Landolt chart was converted into a logarithm of the minimal angle resolution (logMAR) for statistical analysis. The chisquare test was used to compare categorical variables between the two groups. The Mann-Whitney U test was used to compare continuous variables between the two groups. Statistical significance was set at $p<0.05$. 


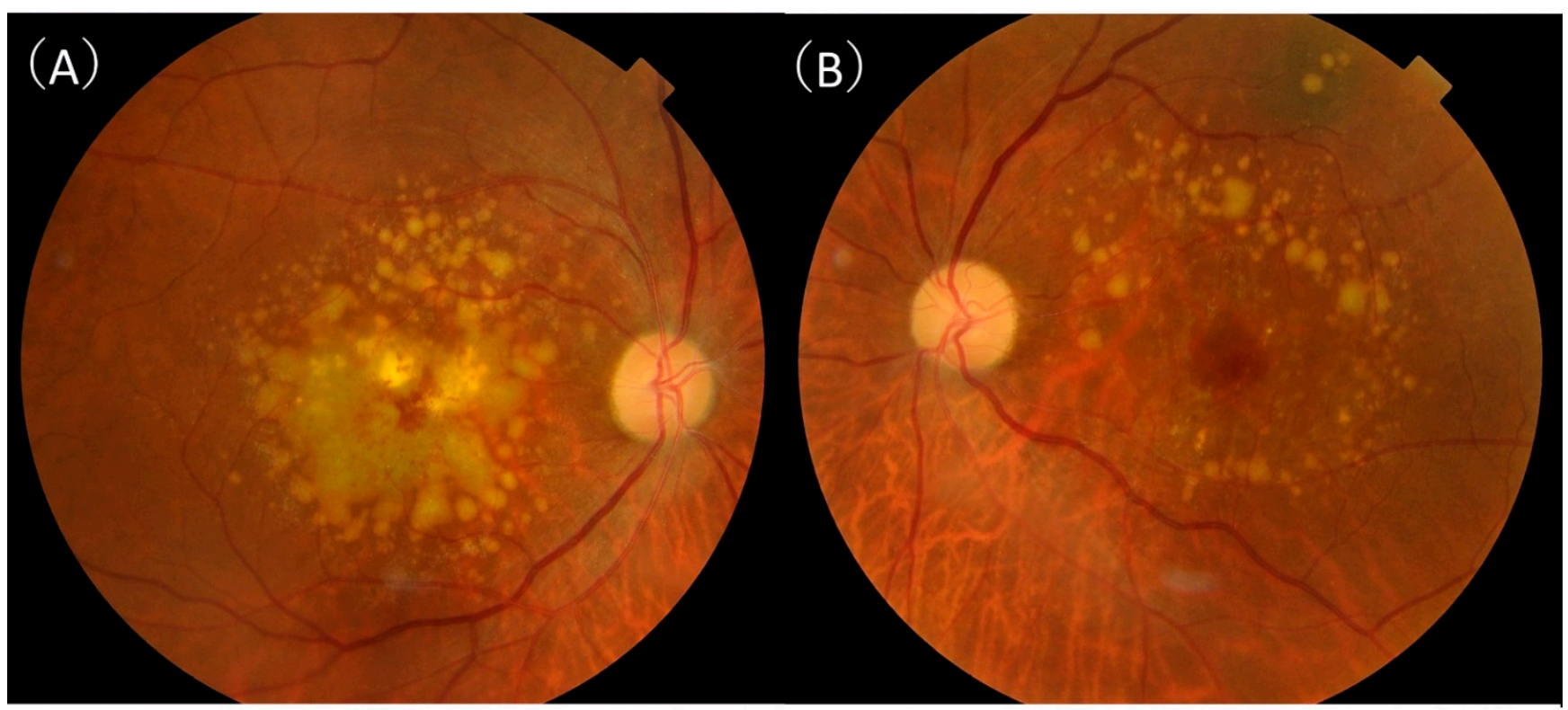

(c)

(D)
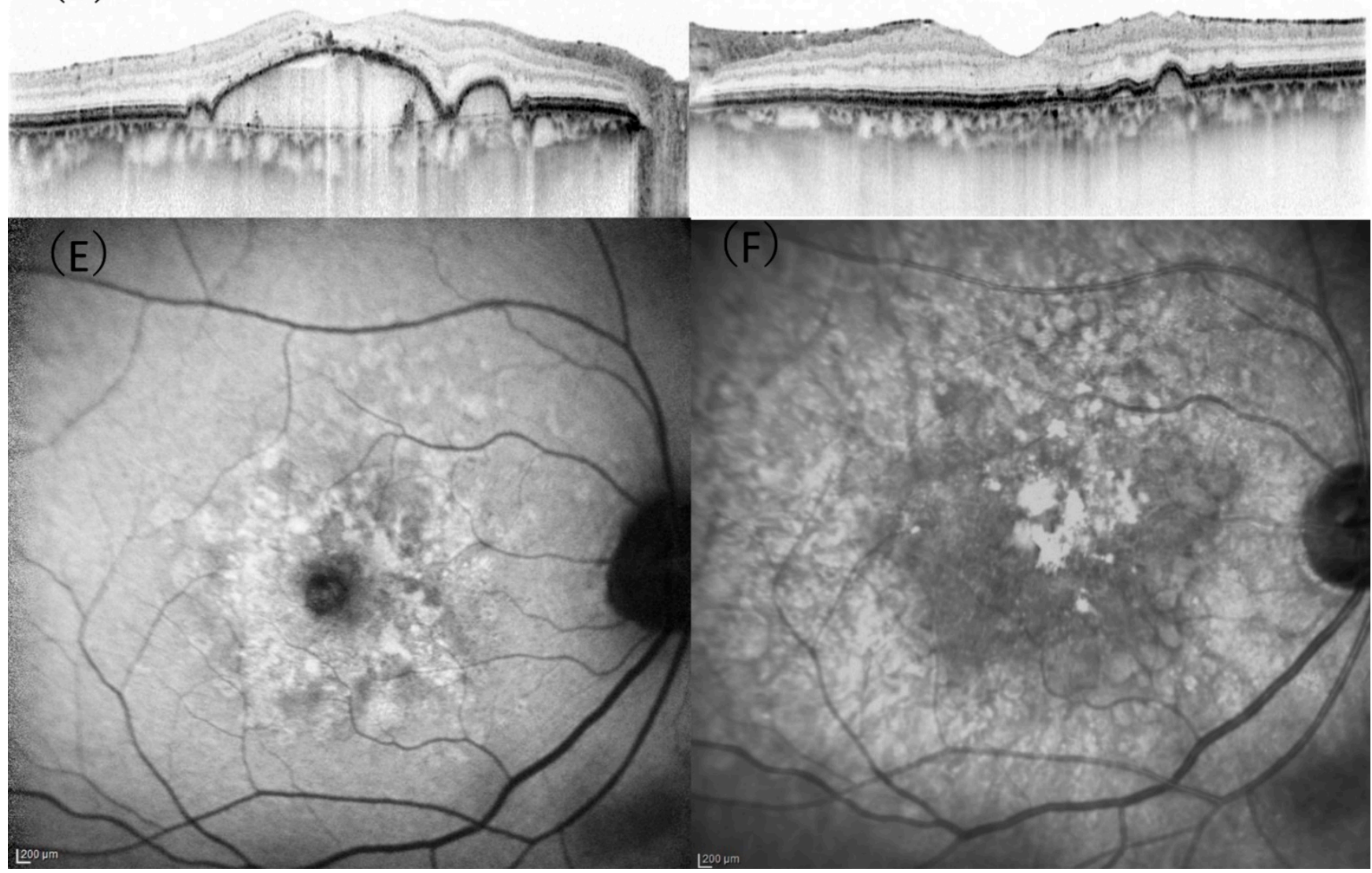

Figure 1. A 75-year-old male patient with drusenoid pigment epithelial detachment. (A) In the right eye, aggregation of confluent drusen was observed in the macula. (B) The aggregation of drusen in the left eye was spared from the central macula. (C) A horizontal optical coherence tomography (OCT) scan showing a large drusenoid pigment epithelial detachment with $307 \mu \mathrm{m}$ height and $3943 \mu \mathrm{m}$ width in the right eye. (D) A horizontal OCT scan showing retinal pigment elevation corresponding to a druse in the left eye. (E) There were no characteristic signs of reticular pseudodrusen on fundus autofluorescence. (F) There were no characteristic signs of reticular pseudodrusen on near-infrared reflectance. 


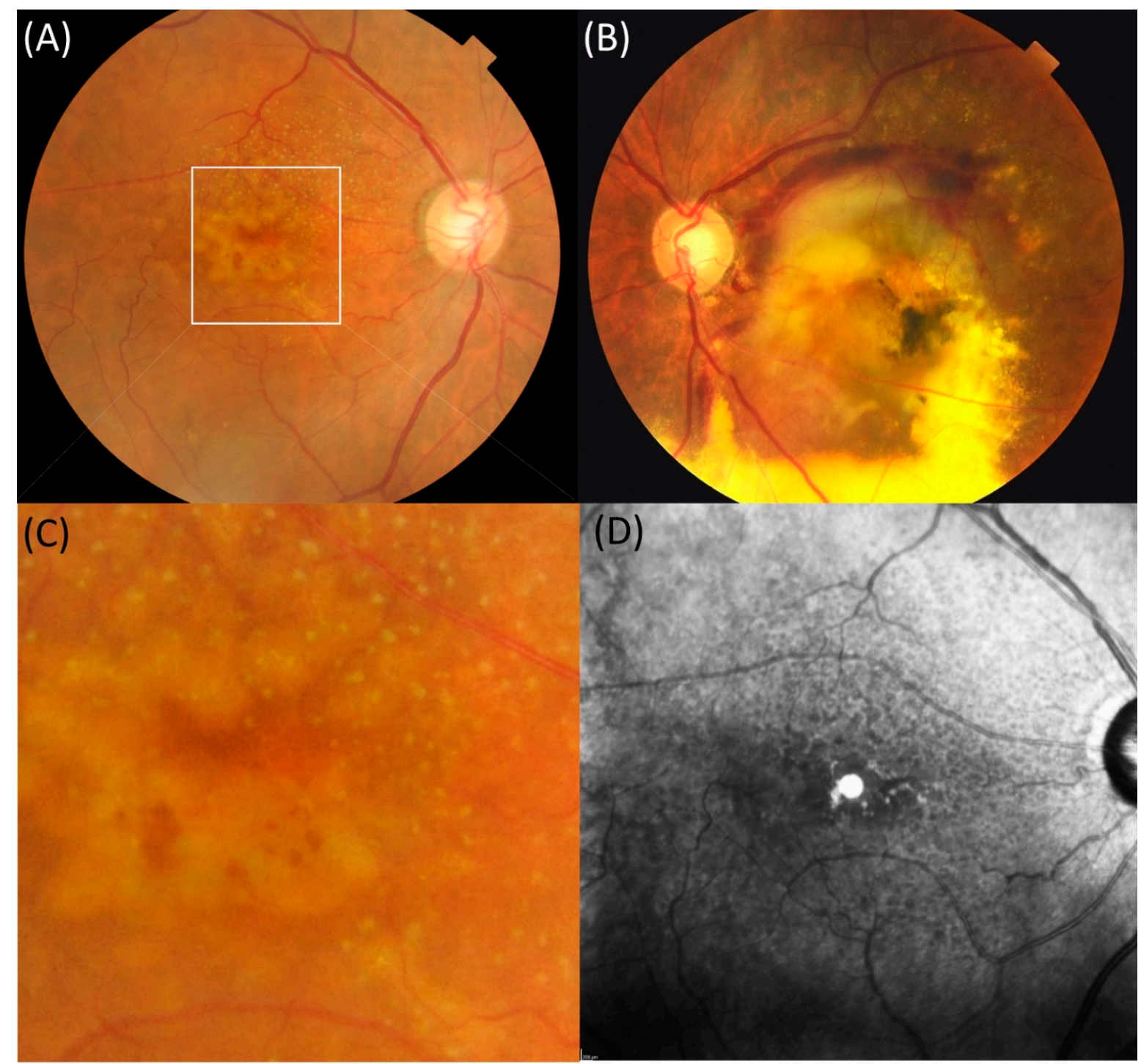

(E)

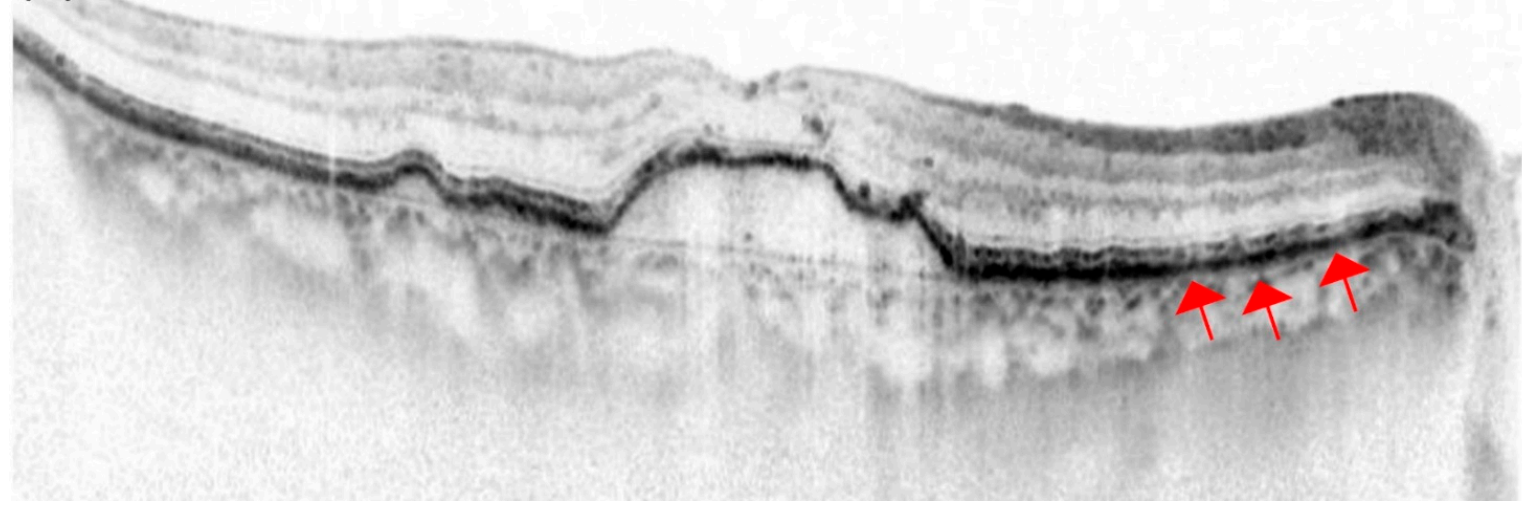

Figure 2. An 83-year-old female patient with drusenoid pigment epithelial detachment showing large subretinal hemorrhage in the contralateral eye. (A) In the right eye, drusen aggregation was observed in the macula. (B) In the left eye, the exudation, mainly subretinal hemorrhage, was seen in the macula. (C) Dot-type reticular pseudodrusen is seen in the magnified image of the macula in the right eye. (D) Hyporeflectance corresponding to the reticular pseudodrusen was mainly observed above the macula in the near-infrared reflectance. (E) Spectral-domain optical coherence tomography (SD-OCT) showed drusenoid pigment epithelial detachment with $113 \mu \mathrm{m}$ height and width of $1872 \mu \mathrm{m}$ in the macula. Red arrows indicate subretinal drusenoid deposits in the retinal pigment epithelium. 
Author Contributions: Concept of the study, Y.S.; Data collection, T.S., Y.S., K.T., A.M., S.Y., Y.M., A.C., Y.W., A.S., H.O., W.K., R.M.; Statistical analysis, T.S.; Writing the original draft, Y.S.; Review and editing of the manuscript, K.T., A.M., R.M.; Supervision, K.K. All authors have read and agreed to the published version of the manuscript.

Funding: This research received no external funding.

Institutional Review Board Statement: This study was approved by Institutional Review Board of University of Yamanashi, Nihon University and Kobe University.

Informed Consent Statement: Informed consent was obtained from all participants involved in the study.

Data Availability Statement: We will provide the data if necessary.

Conflicts of Interest: The authors declare no conflict of interest.

\section{References}

1. Casswell, A.G.; Kohen, D.; Bird, A.C. Retinal pigment epithelial detachments in the elderly: Classification and outcome. Br. J. Ophthalmol. 1985, 69, 397-403. [CrossRef]

2. Balaratnasingam, C.; Yannuzzi, L.A.; Curcio, C.A.; Morgan, W.H.; Querques, G.; Capuano, V.; Souied, E.; Jung, J.; Freund, K.B. Associations Between Retinal Pigment Epithelium and Drusen Volume Changes During the Lifecycle of Large Drusenoid Pigment Epithelial Detachments. Investig. Opthalmol. Vis. Sci. 2016, 57, 5479-5489. [CrossRef]

3. Roquet, W.; Roudot-Thoraval, F.; Coscas, G.; Soubrane, G. Clinical features of drusenoid pigment epithelial detachment in age related macular degeneration. Br. J. Ophthalmol. 2004, 88, 638-642. [CrossRef]

4. Cukras, C.; Agrón, E.; Klein, M.L.; Ferris, F.L., 3rd; Chew, E.Y.; Gensler, G.; Wong, W.T. Natural History of Drusenoid Pigment Epithelial Detachment in Age-Related Macular Degeneration: Age-Related Eye Disease Study Report No. 28. Ophthalmology 2010, 117, 489-499. [CrossRef]

5. Yu, J.J.; Agrón, E.; Clemons, T.E.; Domalpally, A.; Van Asten, F.; Keenan, T.D.; Cukras, C.; Chew, E.Y.; Ferris, F.L.; SanGiovanni, J.P.; et al. Natural History of Drusenoid Pigment Epithelial Detachment Associated with Age-Related Macular Degeneration. Ophthalmology 2019, 126, 261-273. [CrossRef] [PubMed]

6. Cheng, C.-Y.; Yamashiro, K.; Chen, L.J.; Ahn, J.; Huang, L.; Huang, L.; Cheung, C.M.G.; Miyake, M.; Cackett, P.D.; Yeo, I.Y.; et al. New loci and coding variants confer risk for age-related macular degeneration in East Asians. Nat. Commun. 2015, 6, 6063. [CrossRef] [PubMed]

7. $\quad$ Fritsche, L.G.; Igl, W.; Bailey, J.N.C.; Grassmann, F.; Sengupta, S.; Bragg-Gresham, J.L.; Burdon, K.P.; Hebbring, S.J.; Wen, C.; Gorski, M.; et al. A large genome-wide association study of age-related macular degeneration highlights contributions of rare and common variants. Nat. Genet. 2016, 48, 134-143. [CrossRef] [PubMed]

8. Tanaka, K.; Nakayama, T.; Yuzawa, M.; Wang, Z.; Kawamura, A.; Mori, R.; Nakashizuka, H.; Sato, N.; Mizutani, Y. Analysis of candidate genes for age-related macular degeneration subtypes in the Japanese population. Mol. Vis. 2011, 17, 2751-2758. [PubMed]

9. Yoneyama, S.; Sakurada, Y.; Mabuchi, F.; Sugiyama, A.; Kubota, T.; Iijima, H. Genetic Variants in the SKIV2L Gene in Exudative Age-related Macular Degeneration in the Japanese Population. Ophthalmic Genet. 2014, 35, 151-155. [CrossRef] [PubMed]

10. Seddon, J.M.; Francis, P.J.; George, S.; Schultz, D.W.; Rosner, B.; Klein, M.L. Association of CFH Y402H and LOC387715 A69S With Progression of Age-Related Macular Degeneration. JAMA 2007, 297, 1793-1800. [CrossRef]

11. Kim, J.H.; Kang, S.W.; Kim, S.J.; Ha, H.S.; Kim, J.R. Thinner Choroid and Greater Drusen Extent in Retinal Angiomatous Proliferation Than in Typical Exudative Age-Related Macular Degeneration. Am. J. Ophthalmol. 2013, 155, 743-749.e2. [CrossRef]

12. Mimoun, G.; Soubrane, G.; Coscas, G. [Macular drusen]. J. Fr. Ophtalmol. 1990, 13, 511-530. [PubMed]

13. Alten, F.; Clemens, C.R.; Milojcic, C.; Eter, N. Subretinal drusenoid deposits associated with pigment epithelium detachment in age-related macular degeneration. Retina 2012, 32, 1727-1732. [CrossRef]

14. Donato, L.; Scimone, C.; Alibrandi, S.; Pitruzzella, A.; Scalia, F.; D’Angelo, R.; Sidoti, A. Possible A2E Mutagenic Effects on RPE Mitochondrial DNA from Innovative RNA-Seq Bioinformatics Pipeline. Antioxidants 2020, 9, 1158. [CrossRef] [PubMed]

15. Scimone, C.; Alibrandi, S.; Scalinci, S.Z.; Battagliola, E.T.; D’Angelo, R.; Sidoti, A.; Donato, L. Expression of Pro-Angiogenic Markers Is Enhanced by Blue Light in Human RPE Cells. Antioxidants 2020, 9, 1154. [CrossRef] [PubMed]

16. Donato, L.; Scimone, C.; Alibrandi, S.; Abdalla, E.M.; Nabil, K.M.; D’Angelo, R.; Sidoti, A. New Omics-Derived Perspectives on Retinal Dystrophies: Could Ion Channels-Encoding or Related Genes Act as Modifier of Pathological Phenotype? Int. J. Mol. Sci. 2020, 22, 70. [CrossRef] [PubMed]

17. Tan, A.C.; Simhaee, D.; Balaratnasingam, C.; Dansingani, K.K.; Yannuzzi, L.A. A Perspective on the Nature and Frequency of Pigment Epithelial Detachments. Am. J. Ophthalmol. 2016, 172, 13-27. [CrossRef]

18. Sakurada, Y.; Yoneyama, S.; Sugiyama, A.; Tanabe, N.; Kikushima, W.; Mabuchi, F.; Kume, A.; Kubota, T.; Iijima, H. Prevalence and Genetic Characteristics of Geographic Atrophy among Elderly Japanese with Age-Related Macular Degeneration. PLoS ONE 2016, 11, e0149978. [CrossRef] [PubMed] 
19. Shijo, T.; Sakurada, Y.; Yoneyama, S.; Sugiyama, A.; Kikushima, W.; Tanabe, N.; Iijima, H. Prevalence and characteristics of pseudodrusen subtypes in advanced age-related macular degeneration. Graefe's Arch. Clin. Exp. Ophthalmol. 2017, 255, 1125-1131. [CrossRef]

20. Tanaka, K.; Nakayama, T.; Mori, R.; Sato, N.; Kawamura, A.; Mizutani, Y.; Yuzawa, M. Associations of Complement Factor H (CFH) and Age-Related Maculopathy Susceptibility 2 (ARMS2) Genotypes with Subtypes of Polypoidal Choroidal Vasculopathy. Investig. Opthalmol. Vis. Sci. 2011, 52, 7441-7444. [CrossRef]

21. Miki, A.; Honda, S.; Kondo, N.; Negi, A. The Association ofAge-related Maculopathy Susceptibility 2 (ARMS2)andComplement Factor H (CFH)Variants with Two Angiographic Subtypes of Polypoidal Choroidal Vasculopathy. Ophthalmic Genet. 2013, 34, 146-150. [CrossRef] [PubMed]

22. Shijo, T.; Sakurada, Y.; Yoneyama, S.; Kikushima, W.; Sugiyama, A.; Matsubara, M.; Fukuda, Y.; Mabuchi, F.; Kashiwagi, K. Association between Polygenic Risk Score and One-Year Outcomes Following As-Needed Aflibercept Therapy for Exudative Age-Related Macular Degeneration. Pharmaceuticals 2020, 13, 257. [CrossRef] [PubMed] 